\title{
Die Facettenstruktur des Big Five Inventory (BFI)
}

\section{Validierung für die deutsche Adaptation des BFI}

\author{
Beatrice Rammstedt und Daniel Danner
}

\begin{abstract}
Zusammenfassung. Die für die angloamerikanische Version des Big Five Inventory (BFI) entwickelte und validierte Facettenstruktur (Soto \& John, 2009) wurde für die deutsche Adaptation des BFI übertragen und auf ihre Angemessenheit geprüft. Basierend auf drei umfangreichen Stichproben - einer studentischen, einer bildungsheterogenen Stichprobe und einer bevölkerungsrepräsentativen Zufallsstichprobe konnte gezeigt werden, dass die 10 Facetten des deutschen BFI substantielle und mit der angloamerikanischen Version vergleichbare Reliabilitäten und Konvergenzen zwischen Selbst- und Bekanntenurteil und mit den entsprechenden Facetten und Globalskalen des NEOPI-R (NEO-Personality Inventory) und NEO-FFI (NEO-Five Factor Inventory) aufweisen. Ferner konnte eine diskriminante Validität zu den jeweils anderen Facetten der gleichen sowie zu den Facetten der anderen Big Five Dimensionen gezeigt werden. Die Nützlichkeit der Verwendung dieser Facetten, zusätzlich zu den Globalskalen, wurde durch deren spezifische Zusammenhänge mit verschiedenen soziodemografischen und Einstellungsmerkmalen nachgewiesen. Insofern existiert auch für den deutschen Sprachraum ein ökonomisches Maß, um spezifischere Persönlichkeitsaspekte abzubilden.
\end{abstract}

Schlüsselwörter: Persönlichkeitsmessung, Big Five, Facetten, BFI

The Facet Structure of the Big Five Inventory (BFI): Validation for the German Adaptation of the BFI

Abstract. The facet structure (Soto \& John, 2009) developed and validated based on the original US version of the Big Five Inventory (BFI) was applied and validated for the German adaptation of the BFI based on three comprehensive samples: a student sample, a sample heterogeneous with regard to education, and a population-representative random sample. Results indicate that the 10 German BFI facets show substantial (a) reliabilities, (b) convergences between self- and partner ratings, and with the corresponding NEO-PI-R (NEO-Personality Inventory) and NEO-FFI (NEO-Five Factor Inventory) facet and domain scales, as well as (c) discriminant validity within and across domains that are all comparable in size to those reported for the original US version. Differential correlations with several sociodemographic and attitude measures proved the utility of using the BFI facets compared with the domain scales. These BFI facets offer researchers the opportunity to assess efficiently more specific aspects of the Big Five.

Keywords: BFI personality assessment, Big Five, facets, BFI

Im Laufe der letzten Jahrzehnte konnte in der Persönlichkeitsforschung hinsichtlich der Persönlichkeitsstruktur ein breiter Konsensus erzielt werden: Die meisten Forscherinnen und Forscher stimmen dahingehend überein, dass sich die Persönlichkeit auf globaler Ebene durch fünf Dimensionen, die sogenannten Big Five (Goldberg, 1981), beschreiben lässt, nämlich durch Extraversion, Verträglichkeit, Gewissenhaftigkeit, Neurotizismus und Offenheit für Erfahrungen (De Raad, 2000; Goldberg, 1990; John, Naumann \& Soto, 2008). Dieses weitgehende Einvernehmen innerhalb der Persönlichkeitsforschung hat nachhaltig zur Verbesserung der Sichtbarkeit und Akzeptanz von Persönlichkeit generell und speziell der Big Five in anderen wissenschaftlichen Disziplinen beigetragen. Da sich die Big Five für zahlreiche inhaltliche Variablen wie beispielsweise politische Einstellungen und Wahlverhalten (z.B. Vecchione et al., 2011), Gesundheit und Mortalität (z.B.
Roberts, Kuncel, Shiner, Caspi \& Goldberg, 2007) oder Lebenszufriedenheit (z. B. Rammstedt, 2007a) als prädiktiv erwiesen, werden aktuell die Big Five als Indikator für die Persönlichkeit in vielen großangelegten Studien - innerhalb wie außerhalb der Psychologie - miterfasst, so beispielsweise im Sozio-oekonomischen Panel (SOEP), im Nationalen Bildungspanel (NEPS), im Beziehungs- und Familienpanel Panel Analysis of Intimate Relationships and Family Dynamics (pairfam) oder im International Social Survey Programme (ISSP).

Mit den Big Five kann ein breites Spektrum von menschlichem Erleben und Verhalten mit wenigen Dimensionen beschrieben werden. Dies ist einerseits sehr ökonomisch, andererseits geht damit auch ein substantieller Verlust an Informationen einher. Daher wurde vorgeschlagen, Persönlichkeit hierarchisch zu messen (z. B. Cronbach \& Gleser, 1957) und neben globalen Per- 
sönlichkeitsdimensionen ebenfalls spezifische Persönlichkeitsfacetten abzubilden. In den vergangenen Jahren wurde darüber hinaus mehrfach gezeigt, dass im Vergleich zu den Zusammenhängen von inhaltlichen Variablen mit den globalen Big-Five-Dimensionen häufig die spezifischeren Subfaktoren deutliche inkrementelle Validität aufweisen. So zeigten Paunonen und Ashton (2001), dass die spezifischeren Facetten für zahlreiche Verhaltensweisen, wie sportliche Aktivität oder die universitäre Durchschnittsnote, zusätzliche Varianz zu den Big-FiveGlobalskalen erklären konnten. Ähnlich berichten Roberts, Chernyshenko, Stark und Goldberg (2005) für verschiedene Facetten von Gewissenhaftigkeit eine inkrementelle Validität für beispielsweise selbstberichteten Drogenkonsum oder Gesundheitsprävention. Auch scheint sich die Persönlichkeit im Lebensverlauf in verschiedenen Facetten einer Dimension unterschiedlich zu ändern (Soto, John, Gosling \& Potter, 2011). Vor diesem Hintergrund besteht zunehmend ein Bedarf, auch spezifischere Subfaktoren oder Facetten der Big-Five-Persönlichkeitsdimensionen abbilden und erfassen zu können.

Zur Erfassung der Big-Five-Globalskalen existieren zahlreiche Fragebogen (für einen Überblick: z.B. De Raad \& Perugini, 2002). Da die Einsatzfelder für die Erfassung der Big Five stark in ihren Fragestellungen aber auch in ihren Rahmenbedingungen differieren, unterscheiden sich die existierenden Fragebogen deutlich in ihrer Zielsetzung, im Detaillierungsgrad und insbesondere in ihrer Länge. Sehr wenige dieser Fragebogen wurden jedoch in Anlehnung an eine hierarchische Konzeptualisierung der Big-Five entwickelt und erlauben daher, neben der Erfassung der globalen fünf Persönlichkeitsdimensionen, die Erhebung der spezifischeren Subfaktoren oder Facetten. Das bekannteste Big-Five-Instrument, das eine solche hierarchische Struktur aufweist, ist das NEO Personality Inventory in seiner revidierten Fassung (NEO-PI-R, Costa \& McCrae, 1992). Es beinhaltet 240 Items, die die BigFive-Faktoren und pro Faktor sechs Facetten erfassen. Aufgrund dieses Detaillierungsgrads, aber insbesondere auch aufgrund seiner Länge insgesamt, erweist sich das NEO-PI-R für viele Forschungsfragen als zu umfangreich. So sind großangelegte Umfragen, wie das SOEP, NEPS, pairfam oder ISSP, extrem limitiert in ihrer Erhebungszeit. In solchen Erhebungskontexten können somit nur extrem kurze Verfahren zum Einsatz kommen.

Für die Kurzversion des NEO-PI, dem NEO-Five Factor Inventory (NEO-FFI; Costa \& McCrae, 1992), konnte gezeigt werden, dass mit dessen 60 Items 16 der 30 Facetten des NEO-PI-R abgebildet werden können (Chapman, 2007; siehe auch Murray, Rawlings, Allen \& Trinder, 2003;
Saucier, 1998). Eine Problematik des Einsatzes des NEOPI-R oder des NEO-FFI besteht in der Tatsache, dass beide kommerziell vertrieben werden. Forscherinnen und Forscher sind somit genötigt, für jeden erhobenen Fall eine entsprechende Nutzungsgebühr zu zahlen. Daher existiert bislang für Forschungszwecke, in denen weder das NEOPI-R noch der NEO-FFI eingesetzt werden können, keine Möglichkeit, neben den globalen Big Five feingranularere Aspekte dieser Dimensionen abzubilden.

Angesichts dieser Problematik bislang fehlender ökonomischer Erfassungsmöglichkeiten für Facetten der Big Five, entwickelten Soto und John (2009) eine Facettenstruktur für das Big Five Inventory (BFI; John, Donahue \& Kentle, 1991). Diese konstruierten sie auf Basis der 44 Items des BFI und in Anlehnung an die von Costa und McCrae (1992) im Rahmen des NEO-PI-R postulierten Facettenstruktur. Hierzu wurden die BFI-Items jeweils von Expertinnen und Experten hinsichtlich ihrer Facettenzugehörigkeit eingeschätzt und auf Basis empirischer Indikatoren jeweils einer der 30 Facetten des NEO-PI-R zugeordnet. Zehn Facetten ließen sich auf Basis der 44 BFI-Items hinreichend replizieren, je zwei pro Big-FiveDomäne. In einer umfangreichen Validierung, basierend auf einer studentischen sowie einer bevölkerungsheterogenen Gemeindestichprobe, konnten Soto und John (2009) eine ausreichende Reliabilität der Facetten und deren hohe Konvergenz mit den entsprechenden Facetten des NEOPI-R sowie mit Bekanntenurteilen nachweisen.

Die vorliegende Studie hat zum Ziel, die Angemessenheit dieser für die anglo-amerikanische Originalversion des BFI entwickelten Facettenstruktur für die deutsche Adaptation des BFI (Rammstedt, 1997; siehe auch Lang, Lüdtke \& Asendorpf, 2001ํ) zu untersuchen. Ergänzend zur Überprüfung der psychometrischen Qualität der Facetten für den deutschen Sprachraum soll als Erweiterung der Validierungsstudie von Soto und John (2009) überprüft werden, inwieweit die beiden Facetten einer Dimension tatsächlich eine spezifische Validität haben und somit die Erhebung dieser Facetten einen Mehrwert gegenüber den Big-Five-Globalskalen darstellt.

\section{Methode}

\section{Stichproben}

Zur Validierung der Facetten des BFI wurden drei Stichproben, eine studentische, eine bildungs- und altershete-

${ }^{1}$ In beiden Validierungsstudien der deutschen BFI-Adaptationen wurde die Angemessenheit der fünffaktoriellen Struktur konfirmatorisch belegt. 
rogene Stichprobe und eine bevölkerungsrepräsentative Zufallsstichprobe, verwendet. Stichprobe 1 setzte sich aus 453 Personen zusammen (57\% weiblich, fünf Personen machten keine Angaben zu ihrem Geschlecht), wobei die Mehrheit (95\%) Studierende der Universität Bielefeld und Universität Göttingen waren. ${ }^{2}$ Das Alter der Befragten lag bei $M=26(S D=6)$ Jahren.

Stichprobe 2 bestand aus 364 Personen ( $55 \%$ weiblich) im Alter zwischen 15 und 77 Jahren $(M=37, S D=13$ Jahre), die über eine Zeitungsannonce zur Teilnahme an einer persönlichkeitspsychologischen Studie (Andresen, 2002) rekrutiert wurden. Die Stichprobe bestand überwiegend aus Personen mit maximal einem Hauptschul$(n=90)$ oder einem Real- oder Mittelschulabschluss $(n=226)$ und repräsentiert damit annährend die entsprechende Heterogenität in der erwachsenen Bevölkerung. Stichprobe 3 ist eine bevölkerungsrepräsentative Zufallsstichprobe, die im Rahmen der Umfrage "Persönlichkeit und Wahlverhalten" (Schumann, 2004) im Jahre 2003 erhoben wurde und als öffentlicher Datensatz der Wissenschaft über das GESIS-Datenarchiv zur Verfügung steht. Die Stichprobe wurde in einem zweistufigen Verfahren zufällig gezogen. Grundgesamtheit waren in Deutschland lebende Erwachsene (Alter $\geq 18$ Jahre). Die Datenerhebung wurde durch das kommerzielle Erhebungsinstitut Marplan organisiert und durchgeführt. Im Rahmen dieser Umfrage nahmen $N=2508$ Personen an einem 35-minütigen persönlichen Interview teil, in dem primär zentrale demografische Informationen erfasst wurden. Im Anschluss daran erhielten die Befragten eine Fragebogenbatterie, die unter anderem den BFI und den NEO-FFI enthielt. Diese Fragebogenbatterie wurde selbständig von den Befragten bearbeitet und in einem frankierten Rückumschlag zurückgeschickt. Siebenundfünfzig Prozent der initial interviewten Personen sendeten verwertbare Fragebogen zurück. Die Daten dieser $N=1431$ Befragten ( $48 \%$ Frauen; Alter: $M=49, S D=17$, Range: 18-90 Jahre) wurden im Rahmen der vorliegenden Studie analysiert. Eine detaillierte Beschreibung der Stichprobenziehung und der Studiendurchführung findet sich in Schumann (2004). Die Teilnahme an den drei genannten Studien war freiwillig und wurde nicht finanziell honoriert.

\section{Instrumente}

Für sämtliche Befragten der Stichproben 1 und 2 lagen Selbsteinschätzungen im BFI in der Adaptation von Rammstedt (1997) vor. Diese BFI-Adaptation (Rammstedt, 1997) umfasst 45 Items $^{3}$, die auf einer 5-stufigen Likert-Skala von sehr unzutreffend bis sehr zutreffend beantwortet werden. In Anhang A ist die Itemformulierung des BFI dargestellt. Die Adaptation des BFI für den deutschen Sprachraum erfolgte auf Basis einer Übersetzung mit zwei separaten anschließenden Rückübersetzungen und einer integrierenden Rekonziliation, nämlich einer parallelen und integrierenden Diskussion und Abstimmung der beiden Übersetzungsentwürfe. Die psychometrischen Kennwerte erwiesen sich als sehr gut und vollständig vergleichbar zu denen der angloamerikanischen Originalversion (Rammstedt, 1997).

Ein Teil der Stichprobe $1(n=57)$ bearbeitete das BFI nach einem Zeitintervall von 6 Wochen ein zweites Mal. Eine weitere Teilstichprobe der Stichprobe $1(n=158)$ bestand aus heterosexuellen Paaren, die zusätzlich zu den Selbsteinschätzungen auch durch ihre Partnerinnen oder Partner mittels des BFI eingeschätzt wurden, was ermöglicht - ähnlich wie in der angloamerikanischen Validierungsstudie - die Übereinstimmung zwischen Selbstund Bekanntenurteil zu ermitteln. Darüber hinaus bearbeiteten $n=184$ Personen der Stichprobe 1 zusätzlich zum BFI das 240-Item umfassende NEO-PI-R (Costa \& McCrae, 1992; deutsche Adaptation: Ostendorf \& Angleitner, 2004).

Die Befragten der Stichprobe 3 bearbeiteten ebenfalls eine deutsche Version des BFI, allerdings die Adaptation durch Lang et al. (2001). Diese Adaptation basiert auf der ursprünglichen Übersetzung durch Rammstedt und John (siehe Rammstedt, 1997). Elf Items wurden jedoch in ihrer Formulierung leicht verändert (vgl. Lang et al., 2001, für eine Darstellung) und zwei Items ausgeschlossen. ${ }^{4}$ Die Itemformulierungen der resultierenden 42-Item-Version finden sich in Lang et al. (2001). In der vorliegenden Studie wurden die beiden durch Lang et al. (2001) ausgeschlossenen Items in ihrer Adaption durch Rammstedt und John ergänzt: So wurde ergänzend das Verträglichkeitsitem ,sich kooperativ verhält, Zusammenarbeit dem Wettbewerb vorzieht" und das Neurotizismusitem „launisch sein kann, schwankende Stimmungen hat" erfasst,

\footnotetext{
2 Die verbleibenden $5 \%$ der Stichprobe waren keine Studierenden und setzen sich weitestgehend aus Personen aus dem persönlichen Umfeld der Testleiterin und des Testleiters zusammen.

3 Zur Verbesserung der Reliabilität der Skala Verträglichkeit enthält die deutsche Adaptation im Vergleich zu der Originalversion ein zusätzliches Item zur Messung dieser Skala. Dieses Item wurde, da es in der amerikanischen Version nicht existiert, nicht in die Berechnung der Facetten miteinbezogen.

${ }^{4}$ Bei zwei Items führten die Übersetzungsmodifikationen durch Lang et al. (2001) zu einer Bedeutungsveränderung, so dass sie von den Analysen ausgeschlossen und somit nicht Teil der publizierten BFI Version wurden.
} 
so dass insgesamt das BFI mit 44 Items erhoben wurde. Zusätzlich bearbeiteten die Befragten der Stichprobe 3 das NEO-FFI (Costa \& McCrae, 1992; deutsche Adaptation Borkenau \& Ostendorf, 1993). Darüber hinaus beantworteten sie zahlreiche Fragen zu ihrem soziodemografischen Hintergrund und Einstellungsfragen, insbesondere aus dem Bereich der politischen und gesellschaftlichen Einstellungen. Für die vorliegenden Analysen wurden zur Bestimmung der spezifischen Validität folgende soziodemografische Variablen und Einstellungsmaße verwendet (in Klammern jeweils der Variablenname im öffentlich zugänglichen Datensatz): das Alter (alter; kontinuierlich erfasst), das Geschlecht (z15.9; weiblich vs. männlich) und das Bildungsniveau der Befragten (frs16; Schülerin bzw. Schüler, kein Abschluss, Hauptschulabschluss, mittlere Reife, Abitur, abgeschlossenes Studium), Lebenszufriedenheit (fr11; 11-stufige Antwortskala von überhaupt nicht glücklich bis sehr glücklich), Religiosität (frs4; 5-stufige Antwortskala von überhaupt nicht wichtig bis sehr wichtig), politische Wirksamkeit (fr40_13; 5-stufige Antwortskala von trifft überhaupt nicht $z u$ bis trifft voll und ganz zu; invers kodiert) und die Affinität zu einem stabilen kognitiven Orientierungssystem (asko; berechnet aus den binären Items fr7_1-fr7_9; für eine Darstellung der psychometrischen Qualität der Skala siehe Schumann, 2005).

\section{Berechnung der Facetten}

Die zehn Facetten des BFI wurden analog zum von Soto und John (2009) vorgeschlagenen Vorgehen auf Basis der 35 diskriminantesten BFI-Items berechnet. Insbesondere in bevölkerungsheterogenen Stichproben, die sich somit im Mittel - relativ zu den typischen Studierendenstichproben - aus geringer gebildeten Personen zusammensetzen, zeigt sich eine erhöhte Tendenz zu Akquieszenz, also zu der inhaltsunabhängigen Zustimmung zu einem Item. Diese Tendenz überlagert den validen Anteil der Itembeantwortung und beeinflusst somit die psychometrische Qualität der Daten negativ (Rammstedt \& Farmer, 2013; Rammstedt \& Kemper, 2011; Rammstedt, Goldberg \& Borg, 2010; Rammstedt, Kemper \& Borg, 2013). Diese Gefahr besteht insbesondere bei der Verwendung von Instrumenten, die hinsichtlich der Kodierung der Items unbalanciert sind (McCrae, Herbst \& Costa, 2001; Soto, John, Gosling \& Potter, 2008). Da aufgrund der geringen Itemanzahl pro Facette keine vollständige Balancierung der Items erzielt werden konnte, wurde, wie von Soto und John (2009) vorgeschlagen, zunächst für die Tendenz zu Akquieszenz kontrolliert. Hierzu wurde pro Person ein Akquieszenz-Index gebildet: Für diesen wurden die Beantwortungen acht entgegengesetzt gepolter
BFI-Item-Paare gemittelt (z. B. „Ich bin gesprächig, unterhalte mich gerne“ und "Ich bin eher der stille Typ, wortkarg"). Zur Kontrolle der Tendenz zu Akquieszenz wurden die Beantwortungen der BFI-Items jeweils um diesen Index korrigiert (siehe Soto \& John, 2009, Appen$\operatorname{dix} B)$.

Zur Berechnung der Facetten-Scores wurden die um Akquieszenz korrigierten Items pro Facette gemittelt, inhaltlich umgepolte Items wurden vorher rekodiert. Anhang B beschreibt die genaue Zuordnung und Kodierung der Items zur Berechnung der Facetten.

\section{Ergebnisse}

\section{Die Reliabilität der BFI-Facetten}

Zur Bestimmung der Reliabilität der 10 BFI-Facetten wurden separat für die drei Stichproben interne Konsistenzwerte (Cronbachs $\alpha$ ) berechnet. $\mathrm{Zu}$ beachten ist hierbei, dass die einzelnen Facetten lediglich aus zwei bis maximal fünf Items bestehen und insofern vergleichsweise niedrigere Konsistenzen zu erwarten sind. Daher wurde ergänzend die Retest-Korrelation der Facetten basierend auf Daten der studentischen Stichprobe berechnet, da diese weniger von der Itemanzahl abhängig ist. Tabelle 1 zeigt separat für die drei Stichproben die Reliabilitätsschätzer der 10 Facetten der deutschen Adaptation im Vergleich $\mathrm{zu}$ denen der angloamerikanischen Originalversion und im Vergleich zu den entsprechenden Reliabilitäten der BFI-Globalskalen. Im Mittel ergibt sich über alle Facetten hinweg eine interne Konsistenz von $\alpha=.65$ (Range: .43-.82) in der studentischen (Stichprobe 1), von $\alpha=.69$ (Range: .46-.85) in der bildungsheterogenen (Stichprobe 2) und von $\alpha=.62$ (Range: .38-.75) in der Zufallsstichprobe (Stichprobe 3). Die mittleren Konsistenzen der Facetten in der Zufallsstichprobe, die den BFI in der Adaptation von Lang et al. (2001) bearbeitete, fallen somit im Mittel leicht, jedoch signifikant niedriger aus als die der bevölkerungsheterogenen Stichprobe 2, die die Adaptation von Rammstedt und John (siehe Rammstedt, 1997) bearbeitete, $F(363,1430)=1.23, p=$ $.005, \omega^{2}=.04$. Die internen Konsistenzen in Stichprobe 1 und 2 unterscheiden sich im Mittel nicht signifikant voneinander, $F(363,452)=1.16, p=.067, \omega^{2}=.07$, ebenfalls nicht die internen Konsistenzen in Stichprobe 1 und 3, $F(452,1430)=1.06, p=.221, \omega^{2}=.01$. In Stichprobe 1 und 2 weist jeweils die Facette Entgegenkommen und in Stichprobe 3 die Facette Depression die geringsten Konsistenzen auf. Nicht überraschend sind in den drei Stichproben die Konsistenzen der Facetten - insbesondere die 
Tabelle 1. Reliabilitätskoeffizienten und Übereinstimmung zwischen Selbst- und Bekanntenurteil der BFI-Facetten des deutschen BFI im Vergleich zu denen der angloamerikanischen Version

\begin{tabular}{|c|c|c|c|c|c|c|c|c|c|c|}
\hline & \multicolumn{5}{|c|}{ Deutsche Version } & \multicolumn{5}{|c|}{ Angloamerikanische Version } \\
\hline & \multicolumn{3}{|c|}{$\alpha$} & \multirow{2}{*}{$\begin{array}{l}r_{\mathrm{tt}} \\
\mathrm{s} 1\end{array}$} & \multirow{2}{*}{$\begin{array}{l}r_{\mathrm{sb}} \\
\mathrm{s} 1\end{array}$} & \multicolumn{2}{|c|}{$\alpha$} & \multirow{2}{*}{$\begin{array}{c}r_{\mathrm{tt}} \\
\text { StS }\end{array}$} & \multicolumn{2}{|c|}{$r_{s b}$} \\
\hline & s1 & S2 & S3 & & & GS & Sts & & GS & StS \\
\hline \multicolumn{11}{|l|}{ BFI Facette } \\
\hline Durchsetzungsfähigkeit (5) & .82 & .82 & .75 & .91 & .55 & .84 & .83 & .88 & .61 & .63 \\
\hline Aktivität (2) & .62 & .62 & .63 & .77 & .58 & .66 & .76 & .74 & .47 & .54 \\
\hline Altruismus (4) & .62 & .63 & .68 & .71 & .49 & .74 & .68 & .71 & .38 & .44 \\
\hline Entgegenkommen (3) & .43 & .46 & .55 & .70 & .47 & .63 & .61 & .81 & .25 & .45 \\
\hline Ordentlichkeit (2) & .63 & .72 & .51 & .83 & .65 & .63 & .56 & .74 & .48 & .51 \\
\hline Selbstdisziplin (5) & .76 & .75 & .72 & .83 & .48 & .71 & .75 & .77 & .51 & .39 \\
\hline Ängstlichkeit (4) & .79 & .85 & .62 & .74 & .57 & .82 & .83 & .86 & .43 & .51 \\
\hline Depression (2) & .52 & .68 & .38 & .70 & .62 & .65 & .53 & .82 & .44 & .46 \\
\hline Offenheit für Ästhetik (3) & .73 & .80 & .72 & .82 & .66 & .78 & .78 & .82 & .54 & .66 \\
\hline Offenheit für Ideen (5) & .53 & .61 & .68 & .69 & .35 & .72 & .71 & .80 & .36 & .52 \\
\hline Mittelwert & .65 & .69 & .62 & .77 & .54 & .72 & .70 & .80 & .45 & .51 \\
\hline \multicolumn{11}{|l|}{ BFI-Gesamtskala } \\
\hline Extraversion & .88 & .87 & .84 & .93 & .64 & & & & & \\
\hline Verträglichkeit & .75 & .78 & .80 & .74 & .54 & & & & & \\
\hline Gewissenhaftigkeit & .84 & .86 & .84 & .88 & .62 & & & & & \\
\hline Neurotizismus & .86 & .90 & .79 & .72 & .63 & & & & & \\
\hline Offenheit & .75 & .81 & .82 & .81 & .53 & & & & & \\
\hline Mittelwert & .82 & .84 & .82 & .82 & .59 & & & & & \\
\hline
\end{tabular}

Anmerkungen: BFI = Big Five Inventory; $\alpha$ = Cronbachs $\alpha ; r_{\mathrm{tt}}=$ Retest-Korrelation; $r_{\mathrm{sb}}=$ Korrelation zwischen Selbst- und Bekanntenurteil; S = Stichprobe; GS = Gemeindestichprobe, StS = Studentische Stichprobe. Anzahl der Items pro Facette ist in Klammern ergänzt.

der lediglich aus zwei Items bestehenden Facetten Aktivität, Ordentlichkeit und Depression - verglichen mit denen der BFI-Gesamtskalen von im Durchschnitt $\alpha=.82$ bzw. $\alpha=.84$ geringer.

Im Vergleich zu der vorliegenden deutschen Adaptation ergeben sich in den angloamerikanischen Originalskalen leicht höhere Koeffizienten (für die Gemeindestichprobe im Mittel $\alpha=.72 \mathrm{bzw}$. für die angloamerikanische studentische Stichprobe $\alpha=.70$ ), die jedoch in Hinblick auf die Reihung der Facetten mit einer Rangkorrelation von $\rho=.58$ bzw. $\rho=.79$ sehr ähnlich zu denen der deutschen Zufalls(Stichprobe 3) bzw. studentischen Stichprobe (Stichprobe 1) ausfallen. Gemittelt über alle 10 Facetten unterscheiden sich die internen Konsistenzen der deutschen und der amerikanischen Version jedoch weder in den studentischen Stichproben, $F(452,828)=0.83, p=.985, \omega^{2}=0.00$ (Signifikanztest nach Feldt, Woodruff \& Salih, 1987), noch in der bevölkerungsheterogenen Stichprobe, $F(363,641)=$ $0.92, p=.816, \omega^{2}=0.00$.

Über ein Intervall von 6 Wochen weisen die deutschen BFI-Facetten Retest-Korrelationen zwischen $r_{\text {tt }}=.69$ und $r_{\mathrm{tt}}=.91$ mit einem Mittel $^{5}$ von .78 auf und sind damit weitgehend vergleichbar zu den Stabilitäten der BFI-Gesamtskalen mit .83 im Mittel und unterscheiden sich im Durchschnitt nicht signifikant von denen der Originalversion von $.80(z=0.32, p=.749, q=.05)$.

Einschränkend ist anzumerken, dass sowohl in Hinblick auf die interne Konsistenz als auch in Hinblick auf die Retest-Stabilität die aus immerhin fünf Items bestehende Facette Offenheit für Ideen vergleichsweise und bereits kritisch niedrige Werte aufweist (siehe Tabelle 1). Sämtliche Reliabilitätsschätzer der übrigen Facetten hingegen erreichen mindestens einen Wert von .70.

\section{Konstruktvalidität: Übereinstimmung zwischen Selbst- und Bekanntenurteil}

Ein zentraler Indikator für die Konstruktvalidität von Fragebogen stellt die Übereinstimmung zwischen Selbstund Bekanntenberichten mit diesem Instrument dar. Zur

\footnotetext{
${ }^{5}$ Alle Korrelationen wurden vor der Mittelwertbildung Fisher-Z-transformiert.
} 
Ermittlung dieses Indikators für die deutschen BFI-Facetten wurden die Selbsteinschätzungen mit den entsprechenden Einschätzungen durch die Partnerin oder den Partner basierend auf Daten der studentischen Stichprobe korreliert. Diese Korrelationskoeffizienten sind ebenfalls in Tabelle 1 dargestellt, ebenso die entsprechenden bei Soto und John (2009) berichteten Korrelationen zwischen Selbst- und Bekannteneinschätzung für die Facetten des angloamerikanischen BFI. Die Übereinstimmungen zwischen Selbst- und Bekanntenurteil für die deutschen BFIFacetten sind alle von substantiellem Ausmaß mit einem Mittel von $r=.55$. Auffallend ist jedoch wiederum der vergleichsweise geringe Zusammenhang der Facette $O f$ fenheit für Ideen mit $r=.35$. Die Korrelationen zwischen Selbst- und Bekanntenurteil der übrigen neun Facetten variieren zwischen $r=.48$ und $r=.66$. Verglichen mit den entsprechenden Korrelationen für die Gesamtskalen des BFI von $r=.59 \mathrm{im} \mathrm{Mittel,} \mathrm{fallen} \mathrm{die} \mathrm{Konvergenzen} \mathrm{für} \mathrm{die}$ Facetten somit nur geringfügig kleiner aus. Insgesamt sind diese Konvergenzen auch vergleichbar zu den Konvergenzen zwischen den entsprechenden Selbst- und Bekanntenurteilen der amerikanischen studentischen Stichprobe mit einem Mittel von .52. Gemittelt über alle Facetten unterscheiden sich die Korrelationen zwischen der deutschen und der amerikanischen studentischen Stichprobe nicht $(z=0.50, p=.618, q=.04)$.

\section{Die Diskriminanz der BFI-Facetten}

Inwiefern bilden die beiden Facetten pro Big-Five-Dimension tatsächlich unterschiedliche, also diskriminante Aspekte der entsprechenden Dimension ab und inwiefern differieren sie von Aspekten anderer Big-Five-Dimensionen? Die Diskriminanz der 10 BFI-Facetten wurde mittels der Interkorrelationen der Facetten bestimmt. Zusätzlich wurde die Korrelationen der beiden Facetten einer BigFive-Dimension - als Indikator für die Diskriminanz innerhalb einer Domäne - mit den Korrelationen von Facetten unterschiedlicher Dimensionen - als Indikator für die Diskriminanz zwischen Domänen - verglichen.

Tabelle 2 zeigt diese Interkorrelationen der 10 Facetten separat für die drei Stichproben. Wie zu erwarten, ergeben sich mit einer mittleren Korrelation von $r=.52$ in der Zufalls- und mit $r=.51$ in der bildungsheterogenen Stichprobe, bzw. mit $r=.47$ in der studentischen Stichprobe, moderate diskriminante Korrelationen zwischen den Facetten einer Domäne. Diese sind in der Höhe fast identisch zu den von Soto und John (2009) berichteten Koeffizienten von $r=.53$ bzw. $r=.55$. Gemittelt über alle konvergenten Facetten unterscheiden sich die Korrelationen 
zwischen der deutschen und der amerikanischen studentischen Stichprobe nicht $(z \leq 0.78, p \geq .435, q \leq .05)$.

Ebenfalls erwartungskonform fallen die absoluten Korrelationen zwischen Facetten unterschiedlicher Big-FiveDimensionen im Mittel mit $r=.25$ in der Zufallsstichprobe, $r=.14$ in der studentischen und $r=.16$ in der bildungsheterogenen Stichprobe deutlich niedriger aus. Diese sind im Mittel für die studentische und die bildungsheterogene Stichprobe wiederum vergleichbar mit dem für die Originalversion berichteten durchschnittlichen Koeffizienten von $r=.17$ für die studentische und $r=.15$ für die Gemeindestichprobe. Gemittelt über alle divergenten Facetten unterscheiden sich die Korrelationen zwischen der deutschen und der amerikanischen studentischen Stichprobe nicht $(z \leq 0.51, p>.613, q \leq .03)$. Für die Zufallsstichprobe fallen die Interkorrelationen zwar im Mittel höher aus als für die bildungsheterogene Stichprobe, jedoch unterscheiden sich zwischen beiden Stichproben weder die mittleren konvergenten noch die mittleren divergenten Korrelationen signifikant voneinander $(z \leq 1.77, p \geq .076, q \leq .10){ }^{6}$

Zusätzlich wurde die fünffaktorielle Struktur der Facetten mit Explorativen Strukturgleichungsmodellen (ESEM; Asparouhov \& Muthén, 2009) überprüft. Dazu wurde ein Modell mit fünf latenten Faktoren spezifiziert und die Ladungen der 10 manifesten Facetten auf die fünf latenten Faktoren simultan mit dem robusten maximum-likelihood-Algorithmus (MLR) in Mplus 6.1 (Muthén \& Muthén, 1998-2010) geschätzt. Das spezifizierte Modell zeigte in allen drei Stichproben einen guten Fit, root mean square error of approximation (RMSEA) $=.03$, comparative fit in$\operatorname{dex}(\mathrm{CFI})=1.00, \chi^{2}(5)<12.85$. Wie aus den in Tabelle 3 dargestellten standardisierten Faktorladungen ersichtlich, laden erwartungskonform alle Facetten hoch auf den entsprechend konvergenten und niedrig auf den divergenten Faktoren.

\section{Die Konvergenz von BFI- und NEO-PI-R-Facetten}

Die Facetten, basierend auf der angloamerikanischen Originalversion des BFI, wurden konzeptionell in Anlehnung an die von Costa und McCrae (1992) postulierten Facetten des NEO-PI-R entwickelt. Insofern kann für die deutsche
Adaptation eine hohe empirische Übereinstimmung mit den NEO-PI-R-Facetten als ein weiterer Indikator für die Konstruktvalidität der BFI-Facetten angesehen werden. Tabelle 4 stellt - basierend auf den Daten der deutschen studentischen Stichprobe - die Interkorrelationen der 10 BFI-Facetten mit den entsprechenden Facetten des NEOPI-R sowie die mittlere Korrelation mit den fünf divergenten Facetten derselben NEO-PI-R-Dimension dar. Ebenfalls in Tabelle 4 abgetragen sind - basierend auf den Daten sowohl der studentischen als auch der Zufallsstichprobe - die Korrelationen der BFI-Facetten mit den NEO-FFI Globalskalen. ${ }^{7}$ Für sämtliche der 10 Facetten ergeben sich hohe und substantielle Zusammenhänge mit den konvergenten Facetten des NEO-PI-R. Diese variieren zwischen $r=.52$ und $r=.76$ mit einem Mittel von $r=.67$ und sind damit fast identisch in ihrem Ausmaß mit den bei Soto und John (2009) berichteten Konvergenzen von $r=.61 \mathrm{bzw}$. $r=.69 \mathrm{im}$ Mittel $(z=0.46, p=.649, q=.03)$.

Sämtliche der konvergenten Korrelationskoeffizienten zwischen den BFI- und den NEO-PI-R-Facetten sind mit einer mittleren Differenz von .35 maßgeblich höher als die mittlere divergente Korrelation mit den verbleibenden fünf Facetten derselben Big-Five-Dimension. Im Mittel liegt diese divergente Korrelation innerhalb einer Big-Five-Dimension bei $r=.43$ und somit in vergleichbarer Höhe zu der der angloamerikanischen Version mit $r=.44 \mathrm{im}$ Mittel $(z=0.66, p=.508, q=.04)$.

Mit den Globalskalen des NEO-PI-R weisen sämtliche Facetten ebenfalls hohe substantielle Zusammenhänge auf, die zwischen $r=.49$ und $r=.79$ mit einem Mittel von $r=.67$ variieren. Mit einem mittleren $r=.66$ fallen die Korrelationen der BFI-Facetten vergleichbar hoch wie die entsprechenden Globalskalen des NEO-FFI, berechnet auf Basis einer entsprechenden Teilauswertung der NEO-PI-RItems, aus. Basierend auf den Daten der Zufallsstichprobe ergeben sich ebenfalls hohe und substantielle, jedoch im Mittel mit $r=.52$ etwas geringere Zusammenhänge zwischen den BFI-Facetten und den Skalen des NEO-FFI.

\section{Spezifische und inkrementelle Validität der BFI-Facetten}

Eine Facettenstruktur wie beispielsweise die der Big Five dient insbesondere dazu, spezifische Zusammenhänge

\footnotetext{
${ }^{6}$ Die konvergenten und divergenten Korrelationen sind voneinander abhängig, da sie in derselben Stichprobe geschätzt werden. Einige dieser Korrelationen sind abhängig und überlappend (vgl. Meng et al., 1992), andere Korrelationen sind abhängig und nicht überlappend (vgl. Raghunathan, Rosenthal \& Rubin, 1996). Unterschiede in abhängigen, überlappenden Korrelationen und abhängigen, nicht überlappenden Korrelationen können nicht simultan getestet werden. Wir berichten daher die (Fisher-Z-transformierten) gemittelten Korrelationen, die deskriptiv zeigen, dass sich die konvergenten und divergenten Korrelationskoeffizienten bedeutsam voneinander unterscheiden.

Für die studentische Stichprobe wurden die NEO-FFI-Werte basierend auf den Selbsteinschätzungen der 240 NEO-PI-R-Items berechnet.
} 
Tabelle 3. Standardisierte Faktorladungen eines Explorativen Strukturgleichungsmodells (ESEM) mit fünf Faktoren

\begin{tabular}{|c|c|c|c|c|c|}
\hline & $E$ & v & G & $\mathbf{N}$ & 0 \\
\hline \multicolumn{6}{|l|}{ Stichprobe $1(N=453)$} \\
\hline Durchsetzungsfähigkeit & $.67 * * *$ & $-.08 *$ & -.01 & -.11 & -.04 \\
\hline Aktivität & $.95^{\star * *}$ & .01 & .01 & .03 & .03 \\
\hline Altruismus & $.19 *$ & $.45^{* * *}$ & .07 & .05 & .14 \\
\hline Entgegenkommen & -.04 & $.84 * * *$ & -.02 & -.03 & -.02 \\
\hline Ordentlichkeit & -.00 & .02 & $.56 * * *$ & $.13^{\star}$ & $-.17^{\star}$ \\
\hline Selbstdisziplin & -.00 & -.01 & $.99 * * *$ & -.03 & $.03 *$ \\
\hline Ängstlichkeit & .04 & .02 & -.00 & $.85 * * *$ & -.03 \\
\hline Depression & -.19 & -.17 & -.04 & $.57 * * *$ & .07 \\
\hline Offenheit für Ästhetik & -.02 & .03 & -.02 & .06 & $.63^{* * *}$ \\
\hline Offenheit für Ideen & .07 & -.02 & .05 & -.08 & $.60 * * *$ \\
\hline \multicolumn{6}{|l|}{ Stichprobe $2(N=364)$} \\
\hline Durchsetzungsfähigkeit & $1.00 * * *$ & .02 & -.02 & -.00 & -.03 \\
\hline Aktivität & $.46^{* *}$ & $-.16^{\star}$ & $.13^{\star *}$ & $-.25^{\star \star}$ & $.24^{* \star *}$ \\
\hline Altruismus & $.12^{\star}$ & $.69 * * *$ & $.09 *$ & $.13^{*}$ & -.01 \\
\hline Entgegenkommen & -.15 & $.63^{* * *}$ & -.02 & $-.32 * \star \star$ & -.03 \\
\hline Ordentlichkeit & -.05 & .01 & $.80 * * *$ & $.11 *$ & -.08 \\
\hline Selbstdisziplin & .04 & .03 & $.71 * * *$ & -.18 & .09 \\
\hline Ängstlichkeit & -.04 & .05 & -.07 & $.89 * * *$ & -.02 \\
\hline Depression & -.06 & -.14 & .05 & $.73^{* * *}$ & .02 \\
\hline Offenheit für Ästhetik & .02 & .28 & -.03 & $.18 * * *$ & $.56 * * *$ \\
\hline Offenheit für Ideen & -.02 & -.07 & -.01 & -.04 & $.87^{* * *}$ \\
\hline \multicolumn{6}{|l|}{ Stichprobe $3(N=1431)$} \\
\hline Durchsetzungsfähigkeit & $.27 * * *$ & -.01 & $.34^{\star \star *}$ & .03 & 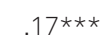 \\
\hline Aktivität & $.85 * * *$ & .01 & .00 & $-.01 * *$ & -.00 \\
\hline Altruismus & $.07 \star \star$ & $.59 * * *$ & -.01 & -.01 & .01 \\
\hline Entgegenkommen & -.06 & $.75^{\star * *}$ & .05 & -.01 & $.02 *$ \\
\hline Ordentlichkeit & -.01 & $.21 * *$ & $.81 * * *$ & $.12^{\star \star \star}$ & $-.09 \star \star \star$ \\
\hline Selbstdisziplin & .01 & $-.03^{* \star}$ & $.67 * * *$ & $-.05^{\star}$ & $.05^{\star * \star}$ \\
\hline Ängstlichkeit & -.03 & .05 & $.06^{*}$ & $.59 * \star *$ & -.01 \\
\hline Depression & .01 & $-.14 * \star \star$ & $-.13 * *$ & $.57^{* * *}$ & .02 \\
\hline Offenheit für Ästhetik & $.09 * *$ & $.20 * * \star$ & -.05 & $.12^{\star \star \star}$ & $.45 * * *$ \\
\hline Offenheit für Ideen & -.01 & .00 & .01 & $-.01 *$ & $.71 * * *$ \\
\hline
\end{tabular}

Anmerkungen: $E=$ Extraversion, $V=$ Verträglichkeit, $G=$ Gewissenhaftigkeit, $N=$ Neurotizismus, $O=$ Offenheit; konvergente Ladungen sind fett gesetzt. ${ }^{* * *} p<.01,{ }^{* *} p<.01,{ }^{*} p<.05, \mathrm{RMSEA}=.03, \mathrm{CFI}=1.00, \chi^{2}(5) \leq 12.85$ in allen Stichproben. Die Residualvarianzen der manifesten Indikatoren wurden in den Stichproben 1 und 3 auf $>0$ restringiert, um Schätzprobleme zu verhindern.

dieser spezifischeren Merkmale mit entsprechenden $\mathrm{Au}-$ ßenkriterien abbilden zu können. Vor diesem Hintergrund ist es essentiell, genau solche Mehrwerte durch eine differentielle und inkrementelle Erklärungskraft der BFI-Facetten nachzuweisen. Auf Basis der Daten der bevölkerungsrepräsentativen Stichprobe 3 wurde daher die spezifische Validität der Facetten des BFI überprüft, indem die beiden Facetten jeder Big-Five-Dimension sowie auch die BFI-Globalskalen mit verschiedenen soziode- mografischen Merkmalen und Einstellungsmaßen korreliert wurden. Zur Überprüfung der inkrementellen Validität wurden die multiplen Korrelationen der demografischen und Einstellungsmaße mit den Big-Five-Globalskalen ohne und mit Einschluss der Facetten berechnet (siehe Tabelle 5). Da insbesondere die politischen Einstellungen stark in Abhängigkeit von der Herkunft variieren, nämlich ob die Person in der ehemaligen BRD, DDR oder im Ausland aufgewachsen ist, wurden in die Analy- 
Tabelle 4. Korrelationen der zehn BFI-Facetten mit den entsprechenden Facetten und Globalskalen des NEO-PI-R und den Globalskalen des NEO-FFI

\begin{tabular}{|c|c|c|c|c|c|}
\hline \multirow[b]{2}{*}{ BFI-Facette } & \multicolumn{3}{|c|}{ NEO-PI-R } & \multicolumn{2}{|c|}{ NEO-FFI } \\
\hline & konvergenteFacette & divergente Facetten & Global & s1 & s3 \\
\hline Durchsetzungsfähigkeit & .64 & .45 & .69 & .59 & .55 \\
\hline Aktivität & .69 & .46 & .72 & .72 & .53 \\
\hline Altruismus & .56 & .33 & .60 & .60 & .54 \\
\hline Entgegenkommen & .52 & .26 & .49 & .54 & .52 \\
\hline Ordentlichkeit & .71 & .37 & .62 & .66 & .48 \\
\hline Selbstdisziplin & .75 & .46 & .74 & .73 & .61 \\
\hline Ängstlichkeit & .76 & .58 & .79 & .75 & .50 \\
\hline Depression & .70 & .57 & .76 & .73 & .46 \\
\hline Offenheit für Ästhetik & .70 & .29 & .56 & .60 & .52 \\
\hline Offenheit für Ideen & .64 & .34 & .61 & .61 & .51 \\
\hline Mittelwert & .67 & .41 & .66 & .65 & .52 \\
\hline
\end{tabular}

Anmerkungen: BFI = Big Five Inventory; NEO-PI-R = NEO Personality Inventory; NEO-FFI = NEO-Five Factor Inventory; S = Stichprobe. Sämtliche berichteten Korrelationskoeffizienten erreichen das Signifikanzniveau von .05.

sen nur Personen eingeschlossen, die angaben, zum Zeitpunkt der Wende in der BRD gelebt zu haben $(83 \%$ der Stichprobe; $n=1219$ ).

Wie aus Tabelle 5 ersichtlich, zeigen sich hinsichtlich der fünf Globalfaktoren die aus bisheriger Forschung bekannten Zusammenhänge: Alter korreliert positiv mit Verträglichkeit und insbesondere Gewissenhaftigkeit, jedoch negativ mit Extraversion (vgl. Feingold, 1994; Goldberg, Sweeney, Merenda \& Hughes, 1998; Herzberg \& Brähler, 2006; Körner, Geyer \& Brähler, 2002; Srivastava, John, Gosling \& Potter, 2002; Rammstedt, 2007b; Roberts et al., 2007; Roberts, Walton \& Viechtbauer, 2006), Frauen weisen im Mittel höhere Verträglichkeits- und Neurotizismuswerte auf (vgl. Costa, Terracciano \& McCrae, 2001; Feingold, 1994; Körner et al., 2002; Rammstedt, 2007b; Srivastava et al., 2002; Viken, Rose, Kapiro \& Koskenvuo, 1994) und Bildung korreliert positiv mit Offenheit (vgl. Caspi, Roberts \& Shiner, 2005; Goldberg et al., 1998; Körner et al., 2002; Vassend \& Skrondal, 1995). In Hinblick auf die Einstellungsmaße replizieren sich die bekannten Korrelationen zwischen Lebenszufriedenheit und Extraversion sowie Neurotizismus (z. B. Diener \& Lucas, 1999); Religiosität weist - wie auch von Paunonen und Jackson (2001) gezeigt - nur geringfügige $\mathrm{Zu}$ sammenhänge mit den Big Five auf, wohingegen Extraversion und Offenheit positiv mit politischer Einflussnahme korrelieren (z.B. Vecchione \& Caprara, 2009). Die in der Studie erfasste Affinität zu einem stabilen kognitiven
Orientierungssystem (ASKO; Schumann, 2001) hat ihre theoretische Fundierung in der Autoritarismusforschung. Ähnlich wie right wing authorianism (RWA; Altemeyer, 1981, 1998; für Zusammenhänge mit den Big Five siehe z. B. Ekehammar, Akrami, Gylje \& Zakrisson, 2004) bestätigen sich auch für die ASKO die negativen Zusammenhänge mit Offenheit und die positiven mit Gewissenhaftigkeit (vgl. Schumann, 2005).

Tabelle 5 zeigt darüber hinaus, dass sich für $\mathrm{Alter}^{8}, \mathrm{Ge}$ schlecht, Bildung, Religiosität und die ASKO differentielle Zusammenhänge mit den BFI-Facetten ergeben. Diese Effekte zeigen sich insbesondere für die beiden Facetten von Offenheit. Ähnlich wie Soto et al. (2011) bereits für das Jugend- und Erwachsenenalter zeigen konnten, variiert Offenheit für Ideen stärker über den Lebensverlauf als $O f$ fenheit für Ästhetik. So nimmt Offenheit für Ideen signifikant stärker mit dem Alter ab als Offenheit für Ästhetik $(z=2.22, p=.026, q=.12$; Berechnung vgl. Meng, Rosenthal \& Rubin, 1992). Frauen berichten eine höhere Offenheit für Ästhetik ( $r=-.16)$, während Männer eher gegenüber Ideen offen sind $(r=.08 ; z=4.44, p<.001, q=.22)$. Auch diese Befunde bestätigen weitgehend die von Soto et al. (2011) berichteten Ergebnisse für das Jugend- und Erwachsenenalter. Religiösere Personen weisen im Mittel eine höhere Offenheit für Ästhetik auf $(r=.11)$, hingegen eine niedrigere Offenheit für Ideen $(r=-.05 ; z=2.94$, $p=.003, q=.16)$. Personen mit einer geringen ASKO sind durchschnittlich offener für Ideen $(r=-.38)$. Der entspre-

\footnotetext{
${ }^{8}$ Da Alter oft keine linearen Zusammenhänge mit der Persönlichkeit zeigt, wurden zusätzlich die Zusammenhänge mit dem quadrierten und dem kubierten Alter untersucht. Die Korrelationskoeffizienten weichen jedoch nicht signifikant voneinander ab $(z \leq 0.13, p \geq .899, q \leq 0.05$; Berechnung vgl. Meng et al., 1992).
} 


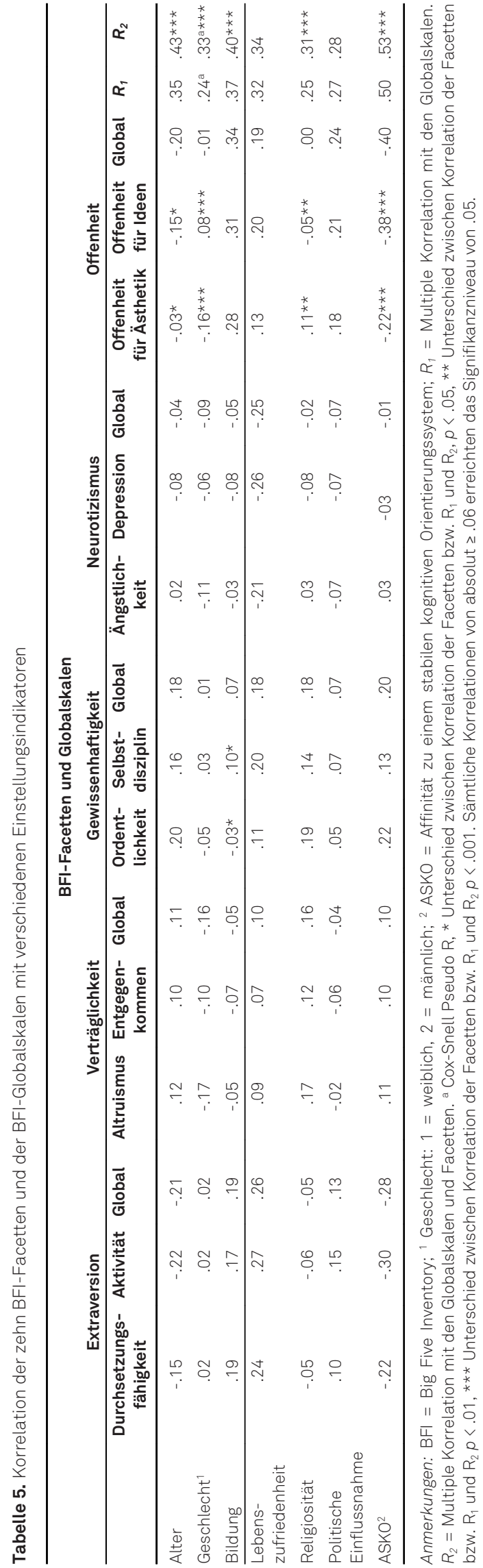

chende Zusammenhang mit Offenheit für Ästhetik weist zwar in dieselbe Richtung, ist vom Ausmaß her jedoch geringer $(r=-.22 ; z=3.34, p<.001, q=.17)$.

Auch in Hinblick auf die Facetten der Gewissenhaftigkeit ergibt sich ein differentieller Befund: Die Facette Selbstdisziplin ist signifikant höher mit Bildung korreliert als die Facette Ordentlichkeit $(z=2.14, p=.033, q=.13)$. Hingegen weist Ordentlichkeit höhere Zusammenhänge mit der ASKO auf als Selbstdisziplin. Die übrigen untersuchten Variablen und Facetten weisen ebenfalls tendentielle, wenn auch nicht signifikante Unterschiede in den Korrelationen auf.

In der Gesamtschau zeigt sich also, dass sich für die hier exemplarisch untersuchten soziodemografischen und Einstellungsvariablen spezifische Zusammenhänge mit den beiden Facetten einer BFI-Dimension ergeben. Darüber hinaus bestätigt sich eine inkrementelle Validität der Facetten gegenüber den Globalskalen: Unter Einbezug der Facetten steigt die multiple Korrelation der demografischen Maße an. Unter Berücksichtigung der Facettenstruktur lassen sich also die vorliegenden untersuchten Merkmale systematisch besser erklären. ${ }^{9}$

Sämtliche der hier dargestellten Befunde wurden auf Basis der Rohwerte repliziert und mit den hier berichteten, um die Tendenz zur Akquieszenz korrigierten Werten verglichen. Es zeigte sich durchgehend ein vergleichbares Muster. Die Koeffizienten wichen nur geringfügig von den hier berichteten ab, fielen jedoch in der Regel leicht geringer aus.

\section{Diskussion}

Soto und John (2009) entwickelten in Anlehnung an die Facetten des NEO-PI-R (Costa \& McCrae, 1992) eine Facettenstruktur für das BFI (John et al., 1991). In diesem hierarchischen Modell wird jede Big-Five-Dimension des BFI mit zwei Facetten abgebildet. Sie konnten nachweisen, dass das BFI nicht nur dann einsetzbar ist, wenn keinerlei Informationen über Facetten benötigt werden (John \& Srivastava, 1999), sondern auch, dass sich vielmehr auf Basis der 44 BFI-Items eine gute Abbildung von zwei unterschiedlichen Merkmalen jeder Dimension identifizieren lassen, die wiederum hohe Übereinstimmungen mit den entsprechenden Facetten des NEO-PI-R aufweisen.

In der vorliegenden Studie wurde die Angemessenheit der für die angloamerikanische Originalversion des BFI entwickelten Facettenstruktur für die deutsche Adaptation des BFI (Lang et al., 2001; Rammstedt, 1997) untersucht und überprüft, inwieweit die beiden Facetten einer 
Dimension tatsächlich eine spezifische und inkrementelle Validität aufweisen und insofern die Erhebung dieser Facetten einen entsprechenden Mehrwert gegenüber den Big-Five-Globalskalen darstellt.

Die psychometrischen Kennwerte der BFI-Facetten wurden mittels dreier unterschiedlicher Stichproben - einer studentischen, einer bildungsheterogenen und einer umfangreichen bevölkerungsrepräsentativen - untersucht. Sowohl die internen Konsistenzen als auch die Retest-Korrelationen als zweites, weniger von der Itemanzahl abhängiges Maß waren für alle Facetten bedeutsam und in ihrer Höhe vergleichbar mit den für die amerikanische Version berichteten Kennwerten.

Zur Bestimmung der Validität der Facetten wurde zum einen die Konvergenz über verschiedene Urteilende (Selbst und Partner bzw. Partnerin) und zum anderen die Konvergenz mit den entsprechenden Facetten des NEOPI-R ermittelt. Ergänzend wurde die divergente Validität bestimmt. Hierzu wurden die Korrelationen der beiden Facetten einer Domäne und die Korrelationen mit Facetten anderer Domänen herangezogen. Die Übereinstimmung zwischen dem Selbst- und Bekanntenurteil ist für die 10 Facetten statistisch bedeutsam und hoch. Darüber hinaus zeigt sich, dass die einzelnen Facetten vergleichbare konvergente Validitäten aufweisen wie (a) die amerikanischen Facetten und (b) die Gesamtskalen das BFI. Auch die konvergenten Korrelationen der BFI-Facetten mit den entsprechenden Facetten des NEO-PI-R sind vergleichbar und vom Ausmaß her fast identisch mit denen der amerikanischen Originalversion. Insofern ist es sowohl der amerikanischen Originalversion in ihrer initialen Entwicklung als auch der Replikation auf Basis der deutschen Adaptation gelungen, die intendierten Konstrukte sehr gut abzubilden.

Um zu überprüfen, ob diese Konstrukte divergent und somit möglichst wenig überlappend sind, wurden die Interkorrelationen der Facetten bestimmt. Diese divergenten Korrelationen, sowohl innerhalb einer Domäne wie auch zwischen Domänen, sind für die Stichproben 1 und 2 wiederum fast identisch zu den bei Soto und John (2009) berichteten Koeffizienten. Leichte Abweichungen in Form höherer divergenter Korrelationen zwischen Domänen ergeben sich allerdings für die Stichprobe 3. Dies kann zum einen in einer unterschiedlichen Stichprobenzusammensetzung begründet sein, zum anderen kann es durch Unterschiede in den verwendeten Instrumenten verursacht sein. In Stichprobe 3 wurde die deutsche BFI-Adaptation von Lang et al. (2001) verwendet, in Stichprobe 1 und 2 die Adaptation durch Rammstedt und John (siehe
Rammstedt, 1997). Auch wenn diese Adaptationen zumeist nur marginal differieren, so unterscheiden sie sich in der Übersetzung des Items Nr. 10, das Teil der Facette Offenheit für Ideen ist. Post-hoc Analysen deuten darauf hin, dass die Adaptation des Items (,erfinderisch und einfallsreich") durch Lang et al. (2001) eine leicht höhere Überlappung mit der Extraversionsfacette Aktivität und hier insbesondere mit dem Item 11 (,voller Energie und Tatendrang") aufweist als die Adaptation von Rammstedt und John (Item 10: „vielseitig interessiert“). Abgesehen von diesem leichten Unterschied in der Divergenz der Facetten und leichten Unterschieden in der internen Konsistenz lässt sich aber konstatieren, dass die Facetten basierend auf den beiden verwendeten deutschen BFIAdaptationen vergleichbare psychometrische Kennwerte aufweisen.

Der Mehrwert der Facetten gegenüber der Verwendung eines Big-Five-Globalmaßes konnte nachgewiesen werden. Sowohl mit klassischen soziodemografischen Merkmalen als auch mit Religiosität wiesen die Facetten spezifische, teils sogar inverse Zusammenhänge auf. Diese Befunde untermauern die Nützlichkeit dieser feingranularen Facettenebene für die Beschreibung und Erklärung von Zusammenhängen.

Diese spezifischen Zusammenhänge können jedoch zunächst nur als ein erster Hinweis auf die inkrementelle Validität der Facetten gegenüber den Globalskalen betrachtet werden. In diesem Licht erscheint es besonders vielversprechend, in künftigen Untersuchungen nicht nur die globalen Persönlichkeitsdimensionen, sondern auch die spezifischen Persönlichkeitsfacetten zu betrachten, die ohne zusätzliche Kosten oder Aufwände mit dem BFI abgebildet werden können.

Die durchgeführten Analysen ermöglichen darüber hinaus auch ein besseres Verständnis der Persönlichkeitsfacetten, die mit Big-Five-Kurzskalen wie dem BFI-K (Rammstedt \& John, 2005) oder dem BFI-10 (Rammstedt, 2007b) abgebildet werden. Die Items des BFI-K und des BFI-10 sind in der Langversion des BFI enthalten und können daher den einzelnen Persönlichkeitsfacetten zugeordnet werden. So bildet das BFI-10 die Facetten Durchsetzungsfähigkeit, Ängstlichkeit, Selbstdisziplin, Altruismus und Offenheit für Ästhetik mit seinen Items ab und ist daher insbesondere für Fragestellungen geeignet, die sich inhaltlich auf diese Facetten beziehen. Die vorliegenden Analysen zeigen, dass die Facetten in unterschiedlicher Weise mit demografischen und Einstellungsmaßen zusammenhängen. Eine explizite Berücksichtigung der Facettenstruktur bei der Auswahl des Messinstruments ist

\footnotetext{
${ }^{9}$ Da die multiplen Korrelationen die Sparsamkeit des Modells nicht explizit berücksichtigen, kann keine Aussage darüber getroffen werden, ob die Vorhersage effizienter ist.
} 


\section{daher entscheidend für die Aussagekraft von empirischen Untersuchungen.}

Zusammengefasst konnte die vorliegende Studie basierend auf drei umfangreichen und teils bevölkerungsrepräsentativen Stichproben zeigen, dass sich mit der deutschen Adaptation des BFI die von Soto und John (2009) postulierte Facettenstruktur nachweisen lässt und dass deren psychometrische Kennwerte vergleichbar mit denen der angloamerikanischen Originalversion sind. Darüber hinaus konnte basierend auf spezifischen Befunden der Mehrwert dieser Facetten gegenüber den Globalskalen nachgewiesen werden.

\section{Literatur}

Altemeyer, B. (1981). Right-wing authoritarianism. Manitoba: University Press.

Altemeyer, B. (1998). The other "authoritarian personality." Advances in experimental social psychology, 30, 47-92.

Andresen, B. (2002). Das Hamburger Persönlichkeitsinventar (HPI). Göttingen: Hogrefe.

Asparouhov, T. \& Muthén, B. (2009). Exploratory structural equation modeling. Structural Equation Modeling, 16, 397 - 438.

Borkenau, P. \& Ostendorf, F. (1993). NEO-Fünf-Faktoren Inventar (NEO-FFI) nach Costa und McCrae. Göttingen: Hogrefe.

Caspi, A., Roberts, B. W. \& Shiner, R. L. (2005). Personality development: Stability and change. Annual Review of Psychology, $56,453-484$.

Chapman, B. P. (2007). Bandwith and Fidelity on the NEO FiveFactor Inventory: replicability and reliability of Saucier's (1998) item cluster subcomponents. Journal of Personality Assessment, 88, 220-234.

Costa, P. T. \& McCrae, R. R. (1992). Revised NEO Personality Inventory and NEO Five Factor Professional Manual. Odessa, FL: Psychological Assessment Resources.

Costa, P. T., Terracciano, A. \& McCrae, R. R. (2001). Gender differences in personality traits across cultures: Robust and surprising findings. Journal of Personality and Social Psychology, 81, $322-331$.

Cronbach, L. J. \& Gleser, G. C. (1957). Psychological tests and personnel decisions. Urbana, IL: University of Illinois Press.

De Raad, B. (2000). The Big Five personality factors. Seattle, WA: Hogrefe.

De Raad, B. \& Perugini, M. (2002). Big Five Assessment. Ashland, US: Hogrefe \& Huber.

Diener, E. \& Lucas, R. E. (1999). Personality and subjective wellbeing. In D. Kahneman, E. Diener \& N. Schwarz (Eds.), Wellbeing: The foundations of hedonic psychology (pp. 213-229). New York, NY: Russell. Sage Foundation.

Ekehammar, B., Akrami, N., Gylje M. \& Zakrisson, I. (2004). What matters most to prejudice: Big Five personality, Social Dominance Orientation, or Right-Wing Authoritarianism? European Journal of Personality, 18, $463-482$.

Feingold, A. (1994). Gender differences in personality - a metaanalysis. Psychological Bulletin, 116, 429- 456.

Feldt, L. S., Woodruff, D. J. \& Salih, F. A. (1987). Statistical inference for coefficient alpha. Applied Psychological Measurement, 11, $93-103$.

Goldberg, L. R. (1981). Language and individual differences: The search for universals in personality lexicons. In L. Wheeler (Ed.),
Review of Personality and Social Psychology (S. 141-165). Beverly Hills, CA: Sage.

Goldberg, L. R. (1990). An alternative "description of personality": The Big-Five factor structure. Journal of Personality and Social Psychology, 59, 1216-1229.

Goldberg, L. R., Sweeney, D., Merenda, P. F. \& Hughes, J. E. (1998). Demographic variables and personality: The effects of gender, age, education, and ethnic/racial status on self-descriptions of personality attributes. Personality and Individual Differences, 24, 393- 403.

Herzberg, P. Y. \& Brähler, E. (2006). Assessing the Big-Five personality domains via short forms. A cautionary note and a proposal. European Journal of Psychological Assessment, 22, 139 148.

John, O. P., Donahue, E. M. \& Kentle, R. L. (1991). The Big Five Inventory - versions $4 a$ and 5. Berkeley, CA: University of California, Berkeley, Institute of Personality and Social Research.

John, O. P., Naumann, L. P. \& Soto, C. J. (2008). Paradigm shift to the integrative Big-Five trait taxonomy: History, measurement, and conceptual issues. In O. P. John, R. W. Robins \& L. A. Pervin (Eds.), Handbook of personality: Theory and research (S. 114158). New York, NY: Guilford Press.

John, O. P. \& Srivastava, S. (1999). The Big Five trait taxonomy: History, measurement, and theoretical perspectives. In L. A. Pervin \& O. P. John (Eds.), Handbook of personality: Theory and research (pp. 102 -138). New York, NY: Guilford Press.

Körner, A., Geyer, M. \& Brähler, E. (2002). Das NEO-Fünf-Faktoren Inventar (NEO-FFI). Validierung anhand einer deutschen Bevölkerungsstichprobe. Diagnostica, 48, 19-27.

Lang, F. R., Lüdtke, O. \& Asendorpf, J. B. (2001). Testgüte und psychometrische Äquivalenz der deutschen Version des Big Five Inventory $(\mathrm{BFI})$ bei jungen, mittelalten und alten Erwachsenen. Diagnostica, 47, 111-121.

McCrae, R. R., Herbst, J. H. \& Costa, P. T. Jr. (2001). Effects of acquiescence on personality factors structures. In R. Rieman, F. M. Spinath \& F. Ostendorf (Eds.), Personality and temperament: Genetics, evolution, and structure (pp. 217-231). Berlin: Pabst Science Publishers.

Meng, X.-L., Rosenthal, R. \& Rubin, D. B. (1992). Comparing Correlated Correlation Coefficients. Psychological Bulletin, 111, $172-175$.

Murray, G., Rawlings, D., Allen, N. B. \& Trinder, J. (2003). NEO FiveFactor Inventory Scores: psychometric properties in a community sample. Measurement and Evaluation in Counseling and Development, 36, 140-149.

Muthén, L. K. \& Muthén, B. O. (1998-2010). Mplus User's Guide $\left(6^{\text {th }}\right.$ ed.). Los Angeles, CA: Muthén \& Muthén.

Ostendorf, F. \& Angleitner, A. (2004). NEO-Persönlichkeitsinventar nach Costa und McCrae, revidierte Fassung. Göttingen: Hogrefe.

Paunonen, S. V. \& Ashton, M. C. (2001). Big Five factors and facets and the prediction of behavior. Journal of Personality and Social Psychology, 81, 524-539.

Paunonen, S. V. \& Jackson, D. N. (2001). What is beyond the Big Five? Plenty! Journal of Personality, 68, $821-835$.

Raghunathan, T. E., Rosenthal, R. \& Rubin, D. B. (1996). Comparing correlated but nonoverlapping correlations. Psychological Methods, 1, 178-183.

Rammstedt, B. (1997). Die deutsche Version des Big Five Inventory (BFI): Übersetzung und Validierung eines Fragebogens zur Erfassung des Fünf-Faktoren-Modells der Persönlichkeit. Unveröffentlichte Diplomarbeit. Bielefeld: Universität Bielefeld.

Rammstedt, B. (2007a). Who worries and who is happy? Explaining individual differences in worries and satisfaction by personality. Personality and Individual Differences, 43, 1626-1634.

Rammstedt, B. (2007b). The 10-Item Big Five Inventory (BFI-10): Norm values and investigation of socio-demographic effects 
based on a German population representative sample. European Journal of Psychological Assessment, 23, 193-201.

Rammstedt, B. \& Farmer, R. (2013). The effects of acquiescence on the Big Five and the moderating role of education. Psychological Assessment, 25, 1137-1145.

Rammstedt, B., Goldberg, L. R. \& Borg, I. (2010). The measurement equivalence of Big-Five factor markers for persons with different levels of education. Journal of Research in Personality, 44, $53-61$.

Rammstedt, B. \& John, O. P. (2005). Kurzversion des Big Five Inventory (BFI-K): Entwicklung und Validierung eines ökonomischen Inventars zur Erfassung der fünf Faktoren der Persönlichkeit. Diagnostica, 51, 195-206.

Rammstedt, B. \& Kemper, C. J. (2011). Measurement equivalence of the Big Five: Shedding further light on potential causes of the educational bias. Journal of Research in Personality, 45, $121-$ 125.

Rammstedt, B., Kemper, C. J. \& Borg, I. (2013). Correcting Big Five measurements for acquiescence: An 18-country cross-cultural study with representative samples. European Journal of Personality, 27, 71-81.

Roberts, B. W., Chernyshenko, O. S., Stark, S. \& Goldberg, L. R. (2005). The structure of conscientiousness: An empirical investigation based on seven major personality questionnaires. Personnel Psychology, 58, $103-139$.

Roberts, B. W., Kuncel, N. R., Shiner, R., Caspi, A. \& Goldberg, L. R. (2007). The power of personality: The comparative validity of personality traits, socioeconomic status and cognitive ability for predicting important life outcomes. Perspectives on Psychological Science, 2, 313-345.

Roberts, B. W., Walton, K. E. \& Viechtbauer, W. (2006). Patterns of mean-level change in personality traits across the life course: A meta-analysis of longitudinal studies. Psychological Bulletin, $132(1), 1-25$.

Saucier, G. (1998). Replicable item-cluster subcomponents in the NEO Five-Factor Inventory. Journal of Personality Assessment, $70,263-276$

Schumann, S. (2001). Persönlichkeitsbedingte Einstellungen zu Parteien. Der Einfluß von Persönlichkeitseigenschaften auf Einstellungen zu politischen Parteien. München, Wien: Oldenbourg.

Schumann, S. (2004): Persönlichkeit und Wahlverhalten 2003. GESIS Data Archive, Köln. ZA4052 data file version 1.0.0. doi:10.4232/1.4052

Schumann, S. (2005). Persönlichkeit. Eine vergessene Größe der empirischen Sozialforschung. Wiesbaden: Verlag für Sozialwissenschaften.
Soto, C. J. \& John, O. P. (2009). Ten facet scales for the Big Five Inventory: Convergence with NEO PI-R facets, self-peer agreement, and discriminant validity. Journal of Research in Personality, 43, 84-90.

Soto, C. J., John, O. P., Gosling, S. D. \& Potter, J. (2008). The developmental psychometrics of Big Five self-reports: Acquiescence, factor structure, coherence, and differentiation from ages 10 to 20. Journal of Personality and Social Psychology, 94, $718-737$

Soto, C. J., John, O. P., Gosling, S. D. \& Potter, J. (2011). Age differences in personality traits from 10 to 65: Big Five domains and facets in a large cross-sectional sample. Journal of Personality and Social Psychology, 100, $330-348$.

Srivastava, S., John, O. P., Gosling, S. D. \& Potter, J. (2002). Development of personality in early and middle adulthood: Set like plaster or persistent change? Journal of Personality and Social Psychology, 84, $1041-1053$.

Vassend, O. \& Skrondal, A. (1995). Factor analytic studies of the NEO-Personality-Inventory and the Five-Factor model: The problem of high structural complexity and conceptual indeterminacy. Personality and Individual Differences, 19, 135-147.

Vecchione, M. \& Caprara, G. V. (2009). Personality determinants of political participation: The contribution of traits and self efficacy beliefs. Personality and Individual Differences, 46, 487-492.

Vecchione, M., Schoen, H., González Castro, J. L., Cieciuch, J., Pavlopoulos, V. \& Caprara, G. V. (2011). Personality correlates of party preference: The Big Five in five big European countries. Personality and Individual Differences, 51, 737-742.

Viken, R. J., Rose, R. J., Kapiro, J. \& Koskenvuo, M. (1994). A developmental genetic analysis of adult personality: Extraversion and neuroticism from 18 to 59 years of age. Journal of Personality and Social Psychology, 4, 722-730.

Onlineveröffentlichung: 02.05.2016

\section{Prof. Dr. Beatrice Rammstedt \\ Daniel Danner}

GESIS - Leibniz-Institut für Sozialwissenschaften

Postfach 122155

68072 Mannheim

beatrice.rammstedt@gesis.org 


\title{
Anhang A
}

\section{Persönlichkeitsfragebogen \\ Big Five Inventory, deutsche Adaptation (Rammstedt, 1997)}

\begin{abstract}
Inwieweit treffen die folgenden Aussagen auf Sie persönlich zu? Wählen Sie dazu bitte die entsprechende Kategorie und kreuzen Sie sie rechts neben der jeweiligen Aussage an!
\end{abstract}

Ich ...

\begin{tabular}{|c|c|c|c|}
\hline $\begin{array}{c}\text { sehr } \\
\text { unzutreffend }\end{array}$ & $\begin{array}{c}\text { eher } \\
\text { unzutreffend }\end{array}$ & $\begin{array}{c}\text { weder } \\
\text { zutreffend } \\
\text { noch } \\
\text { unzutreffend }\end{array}$ & $\begin{array}{cc}\text { eher } & \text { sehr } \\
\text { zutreffend } & \text { zutreffend }\end{array}$ \\
\hline
\end{tabular}

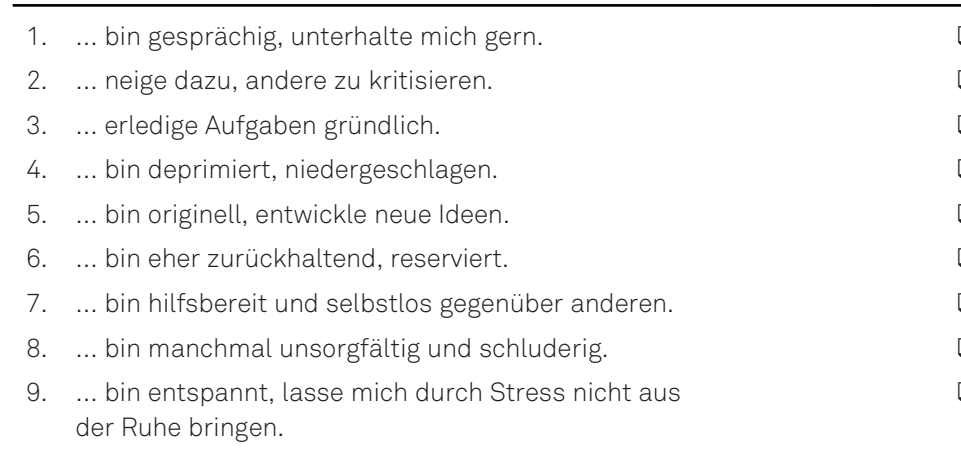

$\begin{array}{lllll}\square & \square & \square & \square & \square \\ \square & \square & \square & \square & \square \\ \square & \square & \square & \square & \square \\ \square & \square & \square & \square & \square \\ \square & \square & \square & \square & \square \\ \square & \square & \square & \square & \square \\ \square & \square & \square & \square & \square \\ \square & \square & \square & \square & \square \\ \square & \square & \square & \square & \square\end{array}$

10. ... bin vielseitig interessiert.

11. ... bin voller Energie und Tatendrang.

12. ... bin häufig in Streitereien verwickelt.

13. ... arbeite zuverlässig und gewissenhaft.

14. ... reagiere leicht angespannt.

15. ... bin tiefsinnig, denke gerne über Sachen nach.

16. ... bin begeisterungsfähig und kann andere leicht mitreißen.

17. ... bin nicht nachtragend, vergebe anderen leicht.

18. ... bin eher unordentlich.

19. ... mache mir viele Sorgen.

20. ... habe eine aktive Vorstellungskraft, bin phantasievoll.

21. ... bin eher der "stille Typ", wortkarg.

22. ... schenke anderen Vertrauen, glaube an das Gute im Menschen.

23. ... bin bequem, neige zur Faulheit.

24. ... bin emotional ausgeglichen, nicht leicht aus der Fassung zu bringen.

25. ... bin erfinderisch und einfallsreich.

26. ... bin durchsetzungsfähig, energisch.

27. ... kann mich kalt und distanziert verhalten.

28. ... harre aus (und arbeite weiter), bis die Aufgabe fertig ist.

29. ... kann launisch sein, habe schwankende Stimmungen.

30. ... schätze künstlerische und ästhetische Eindrücke.

31. ... bin manchmal schüchtern und gehemmt.

32. ... bin rücksichtsvoll zu anderen, einfühlsam.

33. ... bin tüchtig und arbeite flott.

34. ... bleibe ruhig, selbst in Stresssituationen.

35. ... mag es, wenn Aufgaben routinemäßig zu erledigen sind.

36. ... gehe aus mir heraus, bin gesellig. 
Ich ...

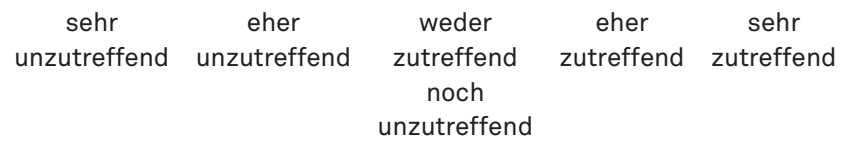

37. ... kann mich schroff und abweisend anderen gegenüber verhalten.

$\begin{array}{lllll}\square & \square & \square & \square & \square \\ \square & \square & \square & \square & \square \\ \square & \square & \square & \square & \square \\ \square & \square & \square & \square & \square \\ \square & \square & \square & \square & \square \\ \square & \square & \square & \square & \square\end{array}$

38. ... mache Pläne und führte sie auch durch.

39. .... werde leicht nervös und unsicher.

40. ... stelle gerne Überlegungen an, spielte mit abstrakten Ideen.

41. ... habe nur wenig künstlerisches Interesse.

42. ... verhalte mich kooperativ, ziehe Zusammenarbeit dem Wettbewerb vor.

43. ... bin leicht ablenkbar, bleibe nicht bei der Sache.

44. ... kenne mich gut in Musik, Kunst oder Literatur aus.

45. ... habe oft Krach mit anderen.

\begin{tabular}{lllll}
$\square$ & $\square$ & $\square$ & $\square$ & $\square$ \\
$\square$ & $\square$ & $\square$ & $\square$ & $\square$ \\
$\square$ & $\square$ & $\square$ & $\square$ & $\square$ \\
\hline
\end{tabular}

\section{Anhang B}

Tabelle B-1. Auswertungsschlüssel zur deutschen Version des BFI

\begin{tabular}{|c|c|c|c|c|}
\hline Extraversion & Verträglichkeit & Gewissenhaftigkeit & Neurotizismus & $\begin{array}{l}\text { Offenheit für } \\
\text { Erfahrungen }\end{array}$ \\
\hline 1 & $2 \mathrm{R}$ & 3 & 4 & 5 \\
\hline $6 \mathrm{R}$ & 7 & $8 \mathrm{R}$ & $9 \mathrm{R}$ & 10 \\
\hline 11 & $12 R$ & 13 & 14 & 15 \\
\hline 16 & 17 & $18 \mathrm{R}$ & 19 & 20 \\
\hline $21 R$ & 22 & $23 R$ & $24 R$ & 25 \\
\hline 26 & $27 R$ & 28 & 29 & 30 \\
\hline $31 \mathrm{R}$ & 32 & 33 & $34 R$ & $35 R$ \\
\hline \multirow[t]{3}{*}{36} & $37 R$ & 38 & 39 & 40 \\
\hline & 42 & $43 R$ & & $41 R$ \\
\hline & $45 R^{a}$ & & & 44 \\
\hline
\end{tabular}

Anmerkungen: $\mathrm{BFI}=$ Big Five Inventory; $\mathrm{R}=$ umgepolte Items. ${ }^{a}$ Item 45 wurde zusätzlich in die deutsche BFI-Version aufgenommen, es ist nicht in der angloamerikanischen Originalversion vorhanden. Es wurde nicht in die Berechnungen im vorliegenden Manuskript inkludiert. Zur Bildung des AkquieszenzIndex werden die in der Tabelle fettgedruckten Items gemittelt (ohne die inhaltlich umgepolten Items vorher zu rekodieren).

Tabelle B-2. Auswertungsschlüssel für die BFI-Facetten

\begin{tabular}{lll}
\hline Big Five-Domäne & BFI-Facette & Items \\
\hline Extraversion & Durchsetzungsfähigkeit & $1,6 \mathrm{R}, 21 \mathrm{R}, 26,31 \mathrm{R}$ \\
Extraversion & Aktivität & 11,16 \\
Verträglichkeit & Altruismus & $7,22,27 \mathrm{R}, 32$ \\
Verträglichkeit & Entgegenkommen & $2 \mathrm{R}, 12 \mathrm{R}, 17$ \\
Gewissenhaftigkeit & Ordentlichkeit & $8 \mathrm{R}, 18 \mathrm{R}$ \\
Gewissenhaftigkeit & Selbstdisziplin & $13,23 \mathrm{R}, 28,38,43 \mathrm{R}$ \\
Neurotizismus & Ängstlichkeit & $9 \mathrm{R}, 19,34 \mathrm{R}, 39$ \\
Neurotizismus & Depression & 4,29 \\
Offenheit & Offenheit für Ästhetik & $30,41 \mathrm{R}, 44$ \\
Offenheit & Offenheit für ldeen & $10,15,25,35 \mathrm{R}, 40$ \\
\hline
\end{tabular}

Anmerkungen: BFI = Big Five Inventory; R = umgepolte Items. 